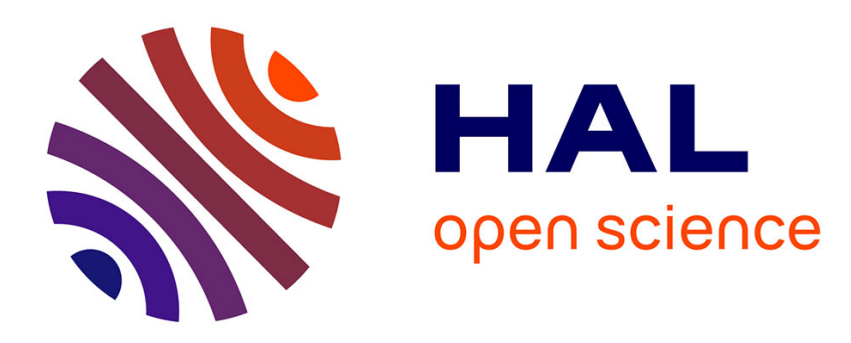

\title{
L'esthétique fictionnelle de Gilbert Lascault
}

Evelyne Toussaint

\section{To cite this version:}

Evelyne Toussaint. L'esthétique fictionnelle de Gilbert Lascault. Critique, 2005, n703, p. 963-973. hal-01884363

\section{HAL Id: hal-01884363 \\ https://hal.science/hal-01884363}

Submitted on 19 Feb 2019

HAL is a multi-disciplinary open access archive for the deposit and dissemination of scientific research documents, whether they are published or not. The documents may come from teaching and research institutions in France or abroad, or from public or private research centers.
L'archive ouverte pluridisciplinaire HAL, est destinée au dépôt et à la diffusion de documents scientifiques de niveau recherche, publiés ou non, émanant des établissements d'enseignement et de recherche français ou étrangers, des laboratoires publics ou privés. 


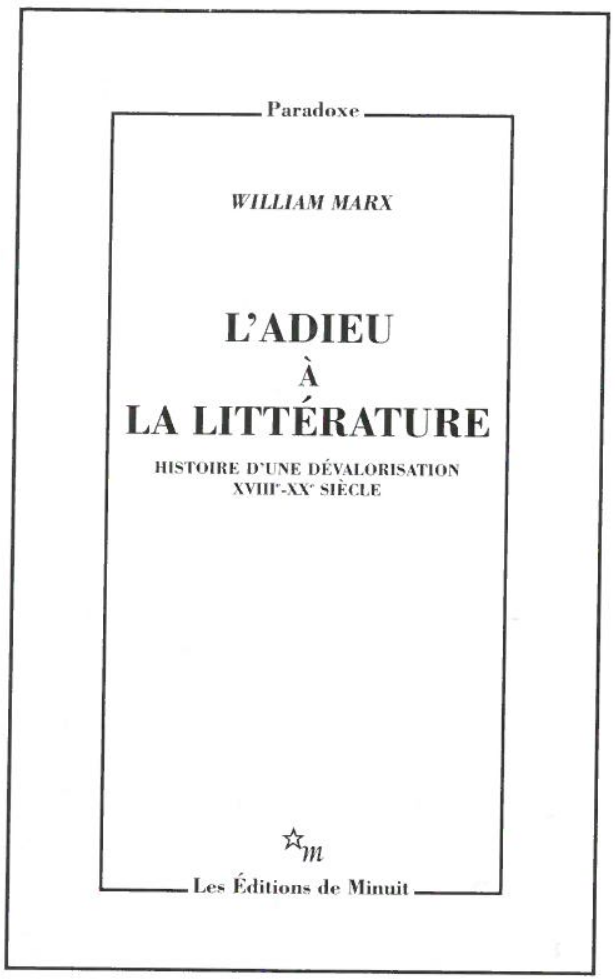

La littérature n'a peut-être jamais été plus mal considérée qu'aujourd'hui. Tous les signes montrent cette fragilisation. Mais plutôt que de s'arrêter à la description d'un mal contemporain dont nul ne doute, ce livre propose de retrouver les causes profondes de cette baisse d'influence, qui résulte d'une évolution de longue durée. La thèse est simple : entre le $x \mathrm{~V}\|\|^{e}$ et le $x x^{e}$ siècle eut lieu en Europe une transformation radicale de la littérature ; sa forme, son idée, sa fonction, sa mission, tout fut bouleversé. Du magnétisme animal aux cultural studies, du sublime selon Boileau au plaisir selon Barthes, du tremblement de terre de Lisbonne au camp d'Auschwitz, de l'apothéose de Voltaire au départ de Rimbaud et aux silences de Beckett, le récit des métamorphoses de la littérature est présenté en une vaste fresque européenne, qui met en evidence un mouvement de bascule conduisant inévitablement du sommet à l'abîme.

Comprendre ce mécanisme de dévalorisation, ce traumatisme de l'adieu, c'est pénétrer au cœur de la crise existentielle permanente où se débat maintenant la littérature. Mais c'est aussi se donner les moyens d'en sortir.

\section{GRITIQUE}

Edmond ORTIGUES

Edelman et la conscience humaine

Laurent ZIMMERMANN

Le temps renversé

Muriel PIC

Les yeux écarquillés :

W. G. Sebald face à la polémique du souvenir

Marie GAILLE-NIKODIMOV Les deux voies de la "biopolitique"

Évelyne TOUSSAINT L'esthétique fictionnelle de Gilbert Lascault

Stéphane BEAU

Plaidoyer pour l'individu

Jean GOLDZINK

Résistances et logique des passions

Tables des matières Tome LXI du n ${ }^{\circ}$ 692/693 au n ${ }^{\circ} 703$

Année 2005 


\section{Sommaire}

Edmond Ortigues : Edelman et la conscience humaine Gerald M. Edelman, Plus vaste est le ciel

Laurent Zimmermann : Le temps renversé

Pierre Bayard, Demain est écrit

Muriel Pic : Les yeux écarquillés. W. G. Sebald face à la polémique du souvenir

Winfried Georg Sebald, De la destruction comme élément de l'histoire naturelle

Marie Gaille-Nikodimov : Les deux voies de la « biopolitique "

Roberto Esposito, Bíos. Biopolitica e filosofia

Évelyne Toussaint : L'esthétique fictionnelle de Gilbert Lascault

Gilbert Lascault, Galaxies amoureuses

$$
\begin{aligned}
& \text { Cartes à jouer et réussites } \\
& \text { Le Monstre dans l'art occidental } \\
& \text { Histoires en forme de trèfle }
\end{aligned}
$$

Les Fables du visible et l'esthétique fictionnelle de Gilbert Lascault (F. Coblence dir.)

Stéphane Beau : Plaidoyer pour l'individu

François de Singly, L'individualisme est un humanisme

Jean Goldzink : Résistances et logique des passions (chez Mme de La Fayette, Prévost, Laclos)

Il y a dix ans disparaissait Jean Piel

Tables des matières de janvier-février 2005 à décembre 2005 Tome LXI du n $692-693$ au n $^{\circ} 703$

DÉCEMBRE 2005

TOME LXI - No 703

publication mensuelle cinquante-huitième année

\section{CRITIQUE \\ Revue générale des publications françaises et étrangères}

Anciens directeurs Georges Bataille, Jean Piel

Comité d'honneur Maurice Blanchot $(\dagger)$, Yves Bonnefoy, Michel Deguy, Jacques Derrida $(\dagger)$, Michel Serres, Jean Starobinski.

\section{Directeur}

PHILIPPE ROGER

Conseil de rédaction Marc Augé, Françoise Balibar, Pierre Birnbaum, Danièle Cohn, Antoine Compagnon, Pedro Cordoba, Élie During, Yves Hersant, Alain de Libera.

Responsable d'édition : Laure Defaction

7, rue Bernard-Palissy - 75006 Paris Tél. : 0144393616 Fax : 0144393615 (le matin seulement)

Les Éditfusion

Philippe Roger reçoit sur rendez-vous.

Les Eninuit

rits ne sont pas retournés.

Les auteurs développent librement une opinion qui n'engage qu'eux-mêmes. 
terrain de l'histoire de la médecine pour aborder le champ de la politique, de considérer le geste immunitaire comme un refus de l'ouverture au devoir envers autrui, on pourra prendre acte des implications de ce geste, y compris en termes de santé. En effet, au cœur de l'analyse étymologique du terme de "communauté" que propose Esposito, se trouve l'idée que les hommes partagent de manière essentielle une dette (le munus de la communauté). Cette analyse ne fait-elle pas écho à la pensée du solidarisme formulée par Léon Bourgeois à la fin du $\mathrm{XX}^{e}$ siècle, principe du système français de la sécurité sociale ${ }^{16}$ ? L'homme "naît débiteur de l'association humaine", car il a besoin de la société pour vivre. L'obéissance au devoir social n'est que la reconnaissance de cette dette de tous contractée avec tous, et c'est par la reconnaissance de celle-ci que la santé de tous est la mieux protégée. Par des biais différents, mais en mettant au centre de leur dispositif théorique l'idée d'une dette, Bourgeois et Esposito parviennent à la même conclusion: la vie humaine est moins bien protégée si l'on se plie au paradigme politique de l'immunité que si on le refuse ${ }^{17}$. C'est toute la pensée politique fondée sur l'idée du contrat, stigmatisée à travers sa figure hobbésienne par Esposito et rousseauiste par Bourgeois, qui est ici mise en question.

\section{Marie GAILLE-NiKodimov}

16. L. Bourgeois, Solidarité, Paris, Colin, 1896

17. Je remercie Frédéric Keck de m'avoir conduite à développer ce point

\section{L'esthétique fictionnelle de Gilbert Lascault}

\section{Gilbert Lascault}

Galaxies amoureuses

Paris, Le Passage, 2004 $237 \mathrm{p}$.

Cartes à jouer et réussites Paris, Bayard, 2003, 127 p.

\author{
Le Monstre \\ dans l'art occidental
}

Paris, Klincksieck, 2004 [1973], $466 \mathrm{p}$.

Histoires en forme de trèfle Avec D. Pouppeville

Paris, Éditions des Lires, 2003 [Seghers, 1990], n.p.

Les Fables du visible et l'esthétique fictionnelle de Gilbert Lascault

F. Coblence (dir.)
Bruxelles, La Lettre volée, 2003, 281 p. "Esthétique fictionnelle" semble un oxymore. Le premier
terme évoque en effet l'étude philosophique et scientifique de l'art, tandis que le caractère imaginaire de la fiction semble la destiner plutôt aux domaines de la littérature ou de la poésie. L'affirmation de l'hétérogène étant peut-être la vraie raison de tout oxymore, l'association des deux mots ne promet nullement une réconciliation des contraires en un tout unifié, mais témoigne à l'inverse d'un désir fondamental de pluralité. Il ne s'agit pas, ici, de délivrer un "bon" savoir sur l'art émanant de 
quelque métalangage, mais de privilégier une méthode vagabonde dont le centre est partout et nulle part: dans la fiction, dans l'érudition, dans la sensation, en utilisant librement nombre de procédés. L'esthétique fictionnelle - possible jalon de cette révolution méthodologique à laquelle Olivier Schefer aspirait récemment ${ }^{1}$ - serait donc un concept empirique, fondé sur des stratégies stylistiques (montage-collage de fragments de discours, décalage par déplacement de l'angle de prise de vue, polysémie): un "concept en quelque sorte", pour reprendre, en les détournant, les termes de Maryvonne Saison, qui évoque l'" esthétique en quelque sorte" de Gilbert Lascault ${ }^{2}$. Sous réserve, bien entendu, de considérer le concept comme réserve infinie de potentialités, non comme clôture, et la méthode qui le sous-tend comme un processus, non comme une règle.

Alors que les chercheurs optent généralement de façon exclusive pour telle ou telle approche théorique (la phénoménologie, ou la philosophie analytique, ou la psychanalyse...), en respectant scrupuleusement les coupures épistémologiques entre esthétique et histoire de l'art, les écrits de Gilbert Lascault défient les certitudes disciplinaires pour prôner l'amour du visible avec, pour seule règle, la volonté d'écarter tout dogmatisme. Affirmant "croire à la méthode mais pas à la théorie", peu convaincu par les outils conceptuels déjà élaborés ${ }^{3}$, Lascault a créé le concept d'esthétique fictionnelle, pervertissant, comme le souligne Francoise Coblence, la division des genres philosophique, littéraire et poétique ( $F V$, p. 10). C'est ce que mettent en lumière Les Fables du visible, ouvrage issu d'un colloque organisé en 2002 à Amiens, mais enrichi de plusieurs contributions - dont deux textes inédits de Gilbert Lascault -, et auquel la participation de vingt-neuf artistes qui ont été sollicités aux fins d'une expérience particulièrement originale donne un caractère

1. O. Schefer, "Un art "gazeux" ou une esthétique éthérée?", Critique, $n^{\circ} 684$, mai 2004, p. 434-436.

2. M. Saison, "L'hybride et le dérisoire, façons de dire à mots couverts", dans Les Fables du visible et l'esthétique fictionnelle de Gilbert

3. do 3ique, comparée, morphologique ou analytique.
gsychologique, sociolo- exceptionnel ${ }^{4}$. Deux rééditions d'œuvres de Gilbert Lascault (Le Monstre dans l'art occidental; Histoires en forme de trèfle) et deux publications récentes (Galaxies amoureuses; Cartes à jouer et réussites) permettent de mettre en perspective un style s'élaborant au fil des écrits, liant art et savoir.

L'esthétique fictionnelle propose ainsi une archéologie des savoirs que l'œuvre contient, mais laisse avant tout place à l'invention, non seulement par l'actualisation que confère à l'objet un nouveau regard, mais encore parce que la fiction, plus que toute herméneutique, est création. Avec la fiction, tout est possible. L'on peut mêler, selon un dispositif intertextuel, descriptions et digressions, narration et érudition, commentaire et invention, sujet de l'énoncé et sujet de l'énonciation. Pour autant, il ne faudrait pas voir là quelque symptôme postmoderniste, une anti-méthode où tout se vaudrait. La fiction n'exclut pas la précision. Gilbert Lascault, qui dit avoir appris de Martial Gueroult l'exigence méthodologique et de Paul Ricœur la conviction que c'est souvent une approche sensible qui répond le mieux aux interrogations philosophiques, invente une poétique de l'esthétique et recherche, après Duchamp, une "exactitude de l'incertain". Ce parti-pris rapprocherait, selon Jacques Duchateau, Lascault de Bachelard, "puisqu'il sait lui aussi rêver une démarche critique si parfaitement rigoureuse".

\section{Le regard et la polysémie}

Daniel Arasse avait dénoncé les écrits érudits qui semblent se passer de regard (On n'y voit rien), ou, au mieux, se contenter d'une vision "de loin" (Le Détail). Certaines descriptions et analyses dissèquent l'œuvre d'art, réduisant, sous prétexte de science, le sensible au savoir. Or l'esthétique fictionnelle de Lascault apprend à voir, parce qu'elle se fonde sur le regard, en incitant d'abord à observer les œuvres de près et de loin, de face et de biais, tantôt trop et tantôt trop peu éclairées, pour "donner à la surprise ses chances", en prenant

4. Une bibliographie exhaustive, établie par Hélène Eristov, fait également de cet ouvrage un précieux outil de référence. Elle recense 9 livres d'esthétique, 29 monographies, 14 fictions, 84 commentaires fictionnels, 230 textes de catalogues, 122 participations à des ouvrages collectifs, ainsi que la création de 23 lithographies. 
la précaution de "se méfier de ce que chacun de nous croit être son désir, et qui n'est souvent que le résultat de ses habitudes, de ses préjugés, de ses petites terreurs injustifiées". Discours et figures se nouent ensuite, après ce temps du regard, démultipliant images et sens.

Dans Le Monstre dans l'art occidental, essai d'esthétique trois fois réédité depuis 1973, Lascault disait vouloir se garder d'"exclure le monstrueux en expliquant le monstre" et s'engageait à ne réduire au discours ni l'art, ni le vivant, ni la sensibilité. Même propos dans tous les écrits ultérieurs, en des jeux de langage qui ne sont pas sans rapport, comme le note Françoise Coblence, avec les modalités du rêve, "présentant les choses sans hiérarchie, les énumérant, les décrivant" ( $F V$, p. 50-51, 55). C'est alors le langage qui se structure comme l'inconscient, selon une logique combinatoire témoignant du goût de l'auteur pour les labyrinthes langagiers, le witz qui advient par condensation, déplacement ou renversement dans le contraire. Contre le réductionnisme de tout quadrillage normatif, il s'agit donc de sauver les effets de scandale, de surprise, de fascination, d'émotion, en empruntant à la méthode psychanalytique qui opère par démembrement et décomposition de chaque élément. Le but est d'instaurer "l'exercice du soupçon", afin de "transgresser les bornes du pensable" en attachant la perception au déni, au désir, à l'angoisse. Quand Ensor déconstruit son propre nom, remarque Gilbert Lascault, il livre, avec ses Squelettes se disputant un hareng, la part tragique de son être (Le Monstre..., p. 369, 337).

Aussi ne viendrait-il jamais à l'idée de Gilbert Lascault de décrire une œuvre selon une grille de lecture systématique. Parfois, comme dans les Histoires en forme de trèfle, il propose un commentaire fictionnel avec l'œuvre. Dans cette réédition de récits, triples virevoltes à partir de six œuvres de Denis Pouppeville, des "divagations" illustrent les images. Tel oiseau s'appelle Arthur et est amoureux des carrés, tel autre aime les rousses opulentes. Dans la Cité où les hauts fonctionnaires sont des chats du Cheshire au sourire énigmatique, chacun est ami de la science et de la volupté, poursuivant des études sur le plaisir, tandis que les assassins du Royaume des Chapeaux pointus élèvent des moustiques de tungstène, les gorgent d'acide, les dressent à tuer et accomplissent ainsi des crimes parfaits.
Quelquefois, pour les Galaxies amoureuses par exemple, Lascault invente des récits à partir de dessins et peintures que nous ne voyons pas, et dont il faut chercher ailleurs la reproduction. Seule constante, dans cette méthode d'une grande plasticité: l'auteur procède par associations d'idées pour inventer des histoires. L'on devine alors comment il a regardé l'image pour qu'advienne le récit, pour nommer les personnages, imaginer l'avant et l'après du moment de la peinture ou du dessin, pour y entrer, y avoir sa place. Comment écrire sur l'art? Regarder, associer des mots aux images, des images aux images et des mots aux mots, mêler narration et érudition, en assujettissant la seconde à la première. Quelquefois l'on pense à Marguerite Duras, par exemple lorsque Lascault, d'après des photographies de Claude Gagean, évoque cette femme qui "désire se fasciner elle-même" et qui "respire, elle"; parfois l'on songe à Raymond Queneau (Gilbert Lascault est, bien sûr, en amitié avec l'Oulipo), car chaque histoire est un exercice de style, un moment du plaisir que l'auteur a pris à regarder peintures, sculptures, estampes, photographies. La fiction concerne aussi bien l'art abstrait, qui "raconte en réalité des histoires qui nous sont très proches", que la figuration, qui "révèle ensuite des problématiques formelles passionnantes" ( $F V$, p. 226), tous les genres et toutes les époques.

L'écriture est un florilège de figures de rhétorique et de jeux polysémiques: allitérations ("Sur un brancard brumeux, le brontosaure bronzé et bronchitique broute un lugubre bréviaire"), oxymorons ("Elle aime les équilibres fragiles et les affolantes impassibilités"), énumérations ("La perspective est déplacée, décalée, flottante, biaisée, gauchie, hors la loi, modifiée, inconstante, capricieuse"), analogies (dans un collage de Joël Kermarrec, "l'oreille du lapin, un creux rose, entouré de fourrure, évoquerait la forme du sexe féminin, celui de la Dame de cœur") et métaphores ("Une roue de la fiction serait de bois vermoulu, rongé", selon un dessin de Daniel Nadaud). Ce choix n'est pas étranger au rythme très particulier des écrits de Lascault, à leur sonorité, à l'effet cinématographique des images et des idées défilant comme autant de plans-séquences (voir Galaxies amoureuses, p. 134, 135, et 228).

Par une autre subtilité stylistique, souvent la méthode mime son objet: "Qui écrit autour du gris ne sera jamais suffi- 
samment absent de son texte. Jamais assez neutre ${ }^{5}$." C'est peut-être au nom du même principe que Gilbert Lascault, fasciné comme Jurgis Baltrusaitis et Henri Focillon par les métamorphoses de formes et de sens, modifie lui-même en permanence ses angles d'approche et s'adonne, avec délectation, à la fantaisie.

Dans les Galaxies amoureuses, chaque œuvre sera à l'origine de ces récits "esquissés, déplacés" qu'affectionne Lascault, proposant des iconologies imaginaires. Telle figure féminine vêtue d'un voile transparent, dans une photographie d'Antoine Poupel, lit peut-être les Contes indiens de Mallarmé. Les êtres des marais dessinés par Henri Cueco ont "l'âme pétrie de boue, de mélancolie et d'humour". Dans les estampes d'Abraham Haddad, les séraphines, les chérubines et les autres angesses recherchent les mortels épris de volupté. Les peintures d'Antonio Seguí évoquent une cité chimérique où les hommes "disent et contredisent", tout en construisant des "demeures qui ne demeurent pas", des "maisons errantes", des "chemins inutiles " (Galaxies amoureuses, p. 191, 152, 219-231). L'esthétique fictionnelle n'a pas pour objet de hiérarchiser les artistes et leurs œuvres, non plus que d'asservir l'œuvre à la théorie. Elle est "à l'opposé des sommations et des injonctions de tous ordres", comme le souligne, avec une gratitude largement partagée, Emmanuel Pernoud. Sa méthode, par conséquent, n'a pas pour objectif de démontrer (Gilbert Lascault proposait à ses étudiants de Nanterre ou de la Sorbonne des "séminaires d'incertitude"), mais d'inventer.

\section{Moi, la fiction, je parle}

Détournant le "moi, la vérité, je parle" de Lacan, Lascault donne, pour Les Fables du Visible, la parole à la fiction: "Moi, la fiction, je parle et je raconte; je bafouille. Je me mêle aux archives. Je me déchire. Je me perds et je me retrouve. Je m'ignore et je m'entrevois. Je me glisse et trouve mes traces. Je circule. Je me réfugie. Je suis multiple et une. Je m'éloigne et je m’approche, dangereusement".

5. G. Lascault, Écrits timides sur le visible, Paris, Armand Colin, 1992 , p. 31.
Instaurant le dialogue, des dessinateurs, peintres, sculpteurs et photographes ${ }^{6}$ avec lesquels Gilbert Lascault a précédemment travaillé lui ont transmis une œuvre où "la fiction agit et parle d'elle". En réponse, Gilbert Lascault parle de la fiction qui parle d'elle. Selon L'Encrier du rêveur de Pierre Alechinsky, la fiction "entrerait dans le Bureau des Sanglots Nocturnes et dans celui des soupirs parfumés". Elle devient "anagramme de Vampire " et "porte un collant noir dans la nuit inquiétante" chez Jean Le Gac. La fiction écoute les Leçons des Ténèbres dans le rayogramme de Christian Boltanski, évoque "les os du thorax du crucifié, à partir du Retable d'Issenheim de Matthias Grünewald" dans les Nielles de Titus-Carmel. Elle mêle "les parfums, les caresses indécises, les baisers légers, les lueurs changeantes" dans une encre de Gilles Ghez.

Et quand la fiction se tait, qui parle? Beaucoup de monde. Dans un écrit publié en 1994, Gens Ordinaires de Sore-lesJardins, le narrateur est "l'ethnologue voilé" et l'on pense à d'autres identités d'emprunt que se sont données les plasticiens contemporains: l'architecte-archéologue inventé par Anne et Patrick Poirier, les alter ego multiples de Raymond Hains (Raymond l'Abstrait, Abstraction personnifiée, Dialecticien des Lapalissades...) et de Jean Le Gac (le Prof de dessin, le Peintre, Lui, Il, Je, Florent Max, Roger Nérac, Ange Glacé, Ramon Nozaro...), le double de Boltanski (le clown Boltanski) ou celui de Sarkis (le capitaine Sarkis). En amitié avec les artistes dont les œuvres inspirent ses récits fictionnels, Gilbert Lascault partage leur aptitude à relativiser un point de vue sur le monde en le modifiant sans cesse. Lui-même se fait un peu Tirésias pour savoir quelque chose de la jouissance féminine, pour en capter tous les voiles et toutes les nudités, tous les territoires et tous les objets, mais après la visite des Galaxies amoureuses, le continent noir garde pourtant ses secrets, car

6. Il s'agit de Pierre Alechinsky, Nicolas Alquin, Christian Boltanski, Pierre Buraglio, Leonardo Cremonini, Henri Cueco, Françoise Dumayet, Claude Gagean, Gilles Ghez, Jean Olivier Hucleux, François Houtin, Christian Jaccard, Joël Kermarrec, Konrad Klapheck, Bernard Lagneau, Alain Lambilliotte, Jean Le Gac, Annette Messager, Ricardo Mosner. Daniel Nadaud, Odon, Anne et Patrick Poirier, Antoine Poupel, Denis Pouppeville, François Rouan, Antonio Segui, Gérard Titus-Carmel et Christian Zeimert 
"la nudité d'une femme est le centre du visible et de l'invisible". Aucune identité d'emprunt ne résoudra le mystère de l'altérité. Heureusement, les ressources de la fiction son inépuisables.

L'indétermination de la place de celui qui parle dans les fictions de Gilbert Lascault confirme encore la position de l'auteur, aux antipodes d'un discours du Pouvoir comme d'un discours du Savoir. L'assurance de n'être maître "ni de moi, ni de l'univers, ni du discours par lequel je voudrais m'affirmer comme tel " éloigne de toute tentation de préjuger autoritairement des intentions des créateurs. Contre certaines convictions de l'histoire de l'art, Gilbert Lascault rappelle que "le désir du créateur [...] n'est jamais transparent à luimême, ni au langage qui prétend l'exprimer" (Le Monstre... p. 180). L'on n'osera donc que des "écrits timides" et de "petites histoires", préférant avec Georges Bataille, comme le souligne Laurence Bertrand Dorléac, "la faiblesse active d'un espoir à jamais l'ennemi de la grande Vérité, totalitaire " (FV p. 17).

Rarement, Gilbert Lascault s'adresse à lui-même: "Tu préfères ne rien espérer, ne pas craindre l'avenir. "L'autobiographie advient ainsi par surprise, dans Cartes à jouer et réussites, avec le souvenir d'un père "silencieux" et "ironique", revenu d'Allemagne en 1945, après cinq années dans un stalag de Prusse orientale. Jouer aux cartes avec lui, c'était "tenter de dialoguer avec réticence". Du coup, ce "décalage" qui fait style dans l'ensemble des écrits résonne affectivement, et l'on perçoit quelque chose des origines de ce dialogue à côté toujours préféré à la frontalité. L'on apprend ainsi que les patiences (comme l'écriture?) "suposent une passion et des actes limités pour résister face au destin " et l'on comprend que les jeux de langages et sourires bienveillants de Gilbert Lascault sont peutêtre, pour partie au moins, une réponse à ce précoce savoir sur la mort (Cartes à jouer..., p. 69 et 88). À cet éclairage, les théâtres de cruauté et les meurtres de 420 minutes dans la cité des ombres et des Marmottes à l'imparfait, dont la violence stupéfiait Pierre Dumayet, se teintent d'autobiographie, comme l'allusion récurrente à La Femme 100 têtes de Max Ernst qui garde, elle aussi, son secret.

\section{Toute fiction est une méthode}

Si l'on veut situer historiquement l'émergence du concept d'esthétique fictionnelle, l'on peut rappeler diverses tentatives "d'écritures alternatives": Michel Butor sur Delvaux, Alain Robbe-Grillet sur Magritte, Jean-François Lyotard sur Monory, Jacques Derrida sur Adami. Aucun de ces auteurs ne s'aviserait de lever l'énigme de l'œuvre, préférant le doute au savoir privilégiant l'indécidable pour donner au lecteur matière à cette "imagination expérimentatrice" qu'affectionnait JeanFrançois Lyotard, parce qu'elle n'a pas à dire vrai au sujet des œuvres, mais à faire œuvre à leur propos. S'interrogeant sur ces écritures alternatives, souvent discontinues et fragmentaires, Anne Mœglin-Delcroix, inversant les termes de Mallarmé - "toute méthode est une fiction" -, constate que "toute fiction est une méthode" ( $F V$, p. 33).

La fiction, dans l'œuvre de Gilbert Lascault, est irréductible à sa transcription en discours univoque. Elle requiert la transversalité, les égarements et les labyrinthes, les analogies et les décentrements, les variations et les anamorphoses. Les références culturelles y prennent place comme dans la distraction, avec une modestie toujours revendiquée, l'auteur s'employant à "penser avec le moins de lourdeur possible " ". Histoire, mythologie, littérature, musique, cinéma, bande dessinée, antiquité et actualité: les savoirs adviennent par fragments. Pour découvrir le monde des Cartes à jouer et réus sites, Gilbert Lascault explore figures et passions de joueurs dans la littérature (Rabelais, Lewis Carroll, Vladimir Nabokov, Claude Lévi-Strauss...), l'art (Georges de La Tour, Chardin, Grandville, les surréalistes, Jean Dubuffet, Cézanne, Léger, Balthus, Dix, le groupe La Seine ${ }^{8} .$. ) ou le cinéma (Raoul Ruiz, Barbet Schroeder). Les couleurs, figures et nombres sont étudiées via Jean Paulhan fasciné par les tarots, Duchamp (les neuf "Moules Mâlics" du Grand Verre seraient des figures de valets), ou Alfred Jarry (dans le jeu de cartes des surréalistes, le Joker est le Père Ubu). Les toiles de Véronique Bigo sont le prétexte, dans les Galaxies amoureuses, à une ébauche d'encyclopédie des images de chaussures dans les contes et les

7. G. Lascault, Boucles et Nœuds, Paris, Balland, 1981, p. 8 .

8. G. Lascault, Porte-folio, Paris, La Seine, 2002. 
peintures, en Chine au $\mathrm{X}^{\mathrm{e}}$ siècle. Chaque commentaire est une fiction érudite où l'on rencontre Baudelaire et Lewis Caroll, Francesco Colonna, Marcel Détienne et Jean-Pierre Vernant, Eschyle, Husserl, Diderot et Derrida, les rituels des Badaga en Inde et les mythes de la Bibliotheca d'Apollodore. Mais toujours l'on se méfiera d'une attitude esthétisante qui évacuerait toute émotion, ainsi que d'une théorie qui exclurait tout humour.

C'est aussi une certaine empathie - souvent jugée discutable par l'esthétique classique, pour cause de non scientificité - qui est ici active, tant avec les créateurs qu'avec leurs créations ("Je suis monstre, ou j'aurais pu l'être, ou peux le devenir", pouvait-on lire dans Le Monstre...). Murielle Gagnebin voit dans les écrits de Gilbert Lascault de "petits romans, bien sûr nourris de savoirs stricts et recherchés, mais avant tout animés du désir de rêver au plus près de l'artiste, avec lui, en sa "compagnie" ( $F V$, p. 222). Le plus souvent la temporalité est celle, suspendue, des contes et légendes, mais dont serait exclu tout déterminisme; ce qui implique l'usage, pour préserver l'imprévisible, du mode conditionnel et des mots ou bien, ou peut-être, ou aussi..., en une "apologie du pluriel". La narration procède par fragments, en boucles et nœuds, dans les marges toujours en passe de basculer, de changer de cap, tout en détours et digressions, elle fonctionne le plus souvent par analogies - comme dans la pensée orientale - ou glacis, en couches légères qui se superposent. Toujours est-il qu'à la fin du parcours l'on se trouve particulièrement éclairé, tout comme lorsque Jacques Derrida déconstruit Adami, ou quand Georges Didi-Huberman chemine avec James Turrell. Mais à dire vrai le parcours ne finit pas: les récits semblent provisoirement interrompus, susceptibles d'être poursuivis. Dans Les Fables $d u$ visible, artistes et chercheurs répondent à leur tour aux fictions de Gilbert Lascault par de nouveaux récits; l'échange pourrait être sans fin, comme celui d'amis qui se raccompagnent mutuellement.

De même, aucune œuvre ne saurait être la dernière, délivrant la vérité ultime de l'art; il n'y a pas de vérité ultime de l'art. C'est pourquoi l'esthétique fictionnelle reste ouverte et déformable. Sa méthode n'ordonne pas, n'obéit pas, ne pontifie pas; elle construit provisoirement. Et puisqu'il y est question de méthode, c'est à un écrit ancien que peut être empruntée la conclusion: "Je crée pour abolir une partie de ma creation. J'invente afin de pouvoir parfois supprimer. Je fais et je défais. Je trace pour effacer et j'efface pour tracer à nouveau. Tantôt j'élève, tantôt j'aplanis, j'égalise. J'aime la métamorphose perpétuelle. Ainsi parle le Mécanicien des Souffles ${ }^{9}$."

Évelyne ToussainT
9. G. Lascault, Courtes légendes (Sur Dominique Labauvie), Paris, Maeght, 1991. 